Apuntes Universitarios, 2021: 11(1), enero-marzo

ISSN: 2304-0335 DOI: https://doi.org/10.17162/au.v11i1.575

\title{
Aprendizaje basado en el proyecto de las especies nativas para la Fitorremediación de las aguas drenadas en la mina Tangana en el distrito de Huachocolpa, Huancavelica
}

\section{Project-based learning of native species for the phytoremediation of drained waters in the Tangana mine in the Huachocolpa District, Huancavelica (Peru)}

\author{
Amadeo Enríquez Donaires, ${ }^{1}$ Luz Marina Acharte Lume, ${ }^{2}$ Daniel Lovera Dávila ${ }^{3}$, \\ Gaby Lili Cabello Santo ${ }^{4}$, Jeny Maribel Asto Gonzales ${ }^{5}$, \\ Jackson Edgardo Perez Carpio ${ }^{6 a}$ \\ Universidad Nacional de Huancavelica, Perú, ${ }^{125}$ \\ Universidad Nacional Mayor de San Marcos, Lima, Perú ${ }^{3}$ \\ Universidad Nacional de Educación Enrique Guzmán y Valle, Lima, Perú ${ }^{4}$ \\ Universidad Peruana Union, Lima, Perú ${ }^{5}$ \\ (iD) ORCID ID: https://orcid.org/0000-0002-8241-0091 ${ }^{1}$ \\ (iD) ORCID ID: https://orcid.org/0000-0001-7717-6408² \\ D ORCID ID: https://orcid.org/ 0000-0003-2815-0716 \\ D ORCID ID: https://orcid.org/0000-0001-9760-3921 \\ D ORCID ID: https://orcid.org/0000-0003-4176-6558 \\ ORCID ID: https://orcid.org/0000-0002-6246-01556
}

Recibido: 12 de enero de 2020

Aceptado: 29 de octubre de 2020

\section{Resumen}

La presente investigación tiene el objetivo de evaluar el aprendizaje basado en el proyecto de las especies nativas para la fitorremediación de las aguas drenadas en la mina Tangana y la capacidad de bioacumulacion de plomo y cadmio mediante las especies putacca (Familia apiaceae) y totora (Scirpus californicus), en el Distrito de Huachocolpa de la Provincia y Departamento de Huancavelica (Perú). Aplicando la metodología analítica instrumental, se obtuvieron resultados satisfactorios para el medio ambiente, pues ambos metales (cadmio y plomo) fueron absorbidos por estas especies. En este contexto, se obtuvieron las siguientes conclusiones: a) La concentración de cadmio (Cd) y plomo $(\mathrm{Pb})$ en aguas drenadas de la mina Tangana fueron $0,2661 \mathrm{mg} \mathrm{Cd} / 1$ y 25,7220 mg Pb/1, la cuantificación de concentración de cadmio $(\mathrm{Cd})$ y plomo $(\mathrm{Pb})$ en la especie totora fue $0,0007 \mathrm{mg} \mathrm{Cd} / \mathrm{Kg}$ y de

\footnotetext{
a Email para correspondecia: jacksonperez@upeu.edu.pe
} 
0,0037mg $\mathrm{Pb} / \mathrm{Kg}$, la cuantificación de concentración de cadmio $(\mathrm{Cd})$ y plomo $(\mathrm{Pb})$ en la especie putacca fue $0,0020 \mathrm{mg} \mathrm{Cd} / \mathrm{Kg}$ y $0,0948 \mathrm{mg} \mathrm{Pb} / \mathrm{Kg}$ ). De lo mencionado se infiere que la putacca posee mayor absorbancia que la totora. b) El aprendizaje colaborativo con el complemento de las TICs permite que los estudiantes de los diversos niveles educativos reduzcan las brechas de acceso a la información e interacción con los expertos en la materia.

Palabras clave: Educación, aprendizaje, proyectos, desarrollo sostenible, fitoremediación, especies nativas, medio ambiente.

\begin{abstract}
The present research aims to evaluate the project-based learning of native species for the phytoremediation of drained waters in the Tangana mine and the bioaccumulation capacity of lead and cadmium through the species putacca (Family apiaceae) and cattail (Scirpus californicus), in the District of Huachocolpa of the Province and Department of Huancavelica (Peru). Applying the instrumental analytical methodology, satisfactory results were obtained for the environment, since both metals (cadmium and lead) were absorbed by these species. In this context, the following conclusions were obtained: a) The concentration of cadmium $(\mathrm{Cd})$ and lead $(\mathrm{Pb})$ in drained waters of the Tangana mine were $0.2661 \mathrm{mg} \mathrm{Cd} / 1$ and $25.7220 \mathrm{mg} \mathrm{Pb} / 1$, the quantification of cadmium $(\mathrm{Cd})$ and lead $(\mathrm{Pb})$ concentration in the cattail species was $0.0007 \mathrm{mg} \mathrm{Cd} / \mathrm{Kg}$ and $0.0037 \mathrm{mg} \mathrm{Pb} / \mathrm{Kg}$, the quantification of the concentration of cadmium $(\mathrm{Cd})$ and lead $(\mathrm{Pb})$ in the putacca species was 0.0020 $\mathrm{mg} \mathrm{Cd} / \mathrm{Kg}$ and $0.0948 \mathrm{mg} \mathrm{Pb} / \mathrm{Kg}$ ). From the above it is inferred that putacca has a higher absorbance than cattail. b) Collaborative learning with the complement of ICTs allows students of different educational levels to reduce the gaps in access to information and interaction with experts in the field.
\end{abstract}

Keywords: Education, learning, projects, sustainable development, phytoremediation, native species, environment.

\title{
Introducción
}

La contaminación del ambiente es uno de los problemas más latentes por la sociedad en que vivimos por la progresiva degradación de los recursos naturales como flora y fauna causada por los drenajes de aguas que salen de la bocamina, conteniendo metales como cadmio y plomo que existen en el lugar de estudio. Por ello, la educación ambiental juega un rol fundamental en la sociedad al respecto, la investigación realizada por Blas y Moreno (2020), menciona que existe una relación e influencia en el aprendizaje como resultado de la educación ambiental con el medio ambiente, teniendo en cuenta la investigación mencionada, se propone el aprendizaje basado en proyectos y la formación integral que están comprometidos con el medio ambiente, estableciendo la interacción con expertos en la materia a fin de conocer, reflexionar en torno a la fitorremediación con plantas nativas y construir sus propuestas educativas de prevención y mitigación.

En este caso, el Ministerio de Educación (MINEDU) en el Perfil del Egreso de Educación 
Básica puntualiza uno de los rasgos, "El estudiante indaga y comprende el mundo natural y artificial utilizando conocimientos científicos en diálogo con saberes locales para mejorar la calidad de vida y el desarrollo sostenible" (MINEDU, 2016). La fitorremediación de suelos contaminados se basa en el uso conjunto de plantas, enmiendas del suelo y técnicas agronómicas para eliminar, retener, o disminuir la toxicidad de los contaminantes del suelo (Carpena y Vidal, 2007).

Cabe resaltar que el plomo es un contaminante mayor en el ambiente y que genera gran preocupación para la salud humana y los ecosistemas Ortiz et al., (2009), así mismo (Rubio et al., 2004), menciona que el plomo es un metal pesados que perjudica la salud de las personas y que para reducir la cantidad de plomo en el suelo se debe realizar estudios de investigación que permitan dar a conocer para dar solución a este problema, a la vez es despachado con aguas residuales y trasportados a los pastizales y cuencas vecinas. El plomo y el cadmio se precipitan en la cadena trófica especialmente en los pastos naturales existentes en el lugar, el consumo de estos pastos produce efectos crónicos en los animales trayendo como consecuencia pérdida económica para los criaderos de la comunidad.

Por otro lado, las especies nativas metalóficas como la putacca (Familia apiaceae) y totora (Scirpus californicus), tienen la capacidad fitorremediadora de absorción de metales pesados como plomo y cadmio, mediante sus raíces y tallos. El presente estudio se basó en el aprendizaje colaborativo y la enseñanza basada en proyectos en torno a la actividad significativa fitorremediadora de las especies nativas como la putacca y totora en aguas drenadas que contienen cadmio y plomo de la bocamina Tangana de la comunidad de Huachocolpa a fin de generar en actuaciones futuras buenas prácticas en la educación ambiental con los estudiantes de la educación básica regular y superior.

\section{Métodos y materiales}

Para lograr el aprendizaje colaborativo en las asignaturas referidas al medio ambiente se investigó las plantas nativas con capacidad de fitorremediación en la enseñanza basada en proyectos a los estudiantes de Educación Básica Regular (EBR) y Educación Superior (ES) empleando el método cualitativo de: sensibilización, indagación y experimentación. Los medios y materiales empleados fueron, videos educativos y observación sistemática en las TICs disponibles para establecer la interacción con los expertos. Para generar en actuaciones futuras en las aulas con los materiales elaborados, para desarrollar buenas prácticas de aprendizaje se consideró el enfoque por competencias y la metodología activa donde el estudiante es el protagonista de la educación y el docente el mediador del aprendizaje. Para lograr los objetivos de la presente investigación utilizamos plantas nativas empleando protocolos de muestreo convenientes. También utilizamos el diseño longitudinal ya que se 
observó la relación entre concentración de cadmio y plomo en las especies nativas (putacca y totora) a través del tiempo.

Los puntos de muestreo de agua en la bocamina fue cada 3 metros hacia adentro, la recolección de la muestra fue en un envase de polietileno de capacidad de 1 litro, debidamente esterilizado y rotulado, realizando el proceso de estabilización con 50\% de Ácido nítrico $\left(\mathrm{HNO}_{3} 1: 1\right)$, para luego llevarlo al laboratorio de Química para su respectivo análisis.

El lugar de muestreo de las especies nativas, se realizó en al Anexo de Pampas Constancia del Distrito de Lircay, obteniendo 1 kilogramo de muestras representativas de cada especie putacca y totora. El proceso de digestión de las muestras de aguas obtenidas cada semana después de la actividad fito-remediadora de las especies putacca y totora, se realizó en un equipo digestor de bloques de Marca Digi PREP SM SCP SCIENCE, de acuerdo con los siguientes pasos:

- 1: Tomar $50 \mathrm{ml}$ de muestra de agua en contacto con la especie nativa previamente agitada.

- 2: Trasvasar al tubo de $50 \mathrm{ml}$,

- 3: Adicionar $2 \mathrm{ml}$ de ácido nítrico $\left(\mathrm{HNO}_{3} 1: 1\right)$ y $1 \mathrm{ml}$ de ácido clorhídrico ( $\left.\mathrm{HCl} 1: 1\right)$

- 4: Colocar al digestor de bloques a una temperatura de $85^{\circ} \mathrm{C}$ durante 3 horas.

- 5: Retirar y enfriar hasta temperatura ambiente y enrasar con agua ultra pura hasta $50 \mathrm{ml}$.

- 6: Tapar los envases y agitar quedando listo para el análisis.

El método utilizado fue mediante la espectrofotometría de Absorción Atómica de flama (Thermo Cientific IC 300 SERIES) ya que esta técnica se basa en la medida de la radiación absorbida por los átomos libres de plomo y cadmio.

\section{Resultados y discusiones}

Según la tabla 1 se puede observar que los valores obtenidos en la concentración del cadmio (Cd) y plomo $(\mathrm{Pb})$, en el agua drenada de la bocamina Tangana fueron 0,2661 mg Cd/L y 25,7220 mg $\mathrm{Pb} / \mathrm{L}$, excedieron la normativa de estándares de calidad de agua por lo que se puede mencionar que está perjudicando el cuerpo receptor. 


\section{Tabla 1}

Resultado de la concentración de Cadmio y Plomo en el agua

\begin{tabular}{lcccc}
\hline Contaminante & Concentración & ECA D.S 004- 2017 MINAM & Categoría 3 & Cumple el ECA \\
\cline { 3 - 3 } & & Riego de vegetales & Bebida de animales & \\
& & $\mathrm{mg} / \mathrm{L}$ & $\mathrm{mg} / \mathrm{L}$ & \\
Cadmio $(\mathrm{Cd})$ & $0,2661 \mathrm{mg} \mathrm{Cd} / \mathrm{L}$ & 0,01 & 0,05 & $\mathrm{NO}$ \\
Plomo $(\mathrm{Pb})$, & $25,7220 \mathrm{mg} \mathrm{Pb} / \mathrm{L}$ & 0,05 & 0,05 & $\mathrm{NO}$ \\
\end{tabular}

Fuente: Propia

Con respecto a los resultados de la concentración de cadmio $(\mathrm{Cd})$ y plomo $(\mathrm{Pb})$ en la especie totora fue $0,0007 \mathrm{mg} \mathrm{Cd} / \mathrm{L}$ y es de $0,0037 \mathrm{mg} \mathrm{Pb} / \mathrm{L}$ y la cuantificación de concentración de cadmio $(\mathrm{Cd})$ y plomo $(\mathrm{Pb})$ en la especie putacca fue $0,0020 \mathrm{mg} \mathrm{Cd} / \mathrm{L}$ y $0,0948 \mathrm{mg} \mathrm{Pb} / \mathrm{L}$ ). Se puede observar que estas dos plantas nativas son bioacomuladora de los metales en estudio de la misma manera (Chaney et al., 1997), menciona que la fitoextración de metales por especies de plantas ofrecen un buen rendimiento para eliminar los metales de suelos.

Es fundamental que los estudiantes conozcan la importancia fitoremediadora de la totora (Scirpus californicus), especie de planta nativa significativamente bioacumuladora de cadmio (Cd) y plomo $(\mathrm{Pb})$, de manera similar la especie putacca (Familia apiaceae), planta nativa significativamente bioacumuladora de cadmio $(\mathrm{Cd})$ y plomo $(\mathrm{Pb})$ en la fitorremediación de las aguas drenadas de la bocamina Tangana.

Según Dale (1986), menciona que el 90\% de lo que se dice y hace corresponde a un aprendizaje activo-colaborativo, basado en proyectos donde se tiene contacto directo con la realidad, es decir el aprendiz desarrolla capacidades de análisis, diseño, creación y evaluación. En ese sentido en el Perú los enfoques transversales definidos en el Currículo Nacional se basan en los principios establecidos en el Artículo $8^{\circ}$ de la Ley General de Educación: calidad, equidad, ética, democracia, conciencia ambiental, interculturalidad, inclusión, creatividad e innovación. Es por ello que es necesario fomentar actividades significativas y proyectos de aprendizaje desde la educación básica para concientizar a las futuras generaciones y mitigar acciones que contaminen el medio ambiente.

El aprendizaje basado en el proyecto es una fuente rica y original que nos brinda información en torno a las especies nativas (putacca y totora) cuyo valor radica en la captura de metales de aguas drenadas de mina, es por ello que consideramos oportuno y necesario que los currículos de formación 
de Educación Básica Regular y Educación Superior actualicen e inserten contenidos en las áreas curriculares de los diferentes niveles educativos a fin de informar y concientizar a los futuros ciudadanos sobre la existencia de estas especies nativas. En la tabla 2 mostramos en que áreas curriculares se requiere actualización e inserción de las especies nativas que capturan metales.

\section{Tabla 2}

Áreas curriculares que requieren actualización e inserción de las especies nativas que capturan metales de aguas drenadas de mina

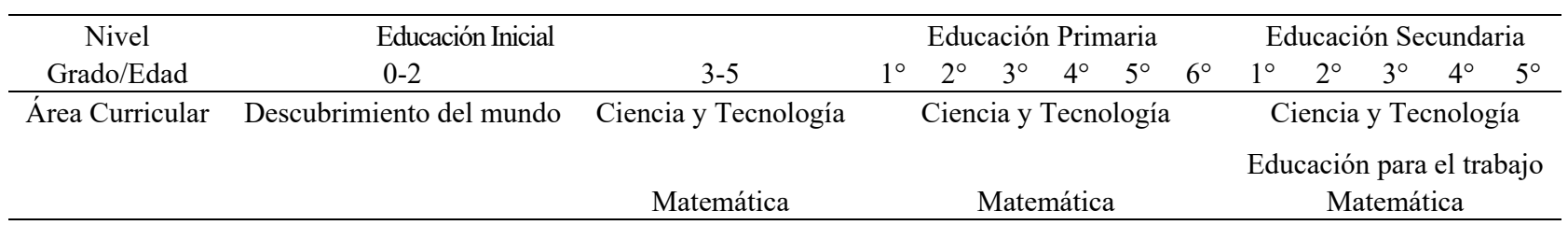

Fuente: MINEDU. (2017). Currículo Nacional de Educación Básica Regular. P.191

En el nivel superior, la Ley Universitaria 30220 establece en su art. 40, "El currículo se debe actualizar cada tres (3) años o cuando sea conveniente, según los avances científicos y tecnológicos”. En consecuencia, amerita la evaluación y renovación en los planes de estudio de las Universidades públicas y privadas. A continuación, se ha tomado tres universidades nacionales del Perú donde se recomienda se inserten contenidos relacionados con las especies nativas que capturan metales de aguas drenadas de mina. Ver tabla 3.

\section{Tabla 3}

Asignaturas que requieren actualización e inserción de las especies nativas que capturan metales de aguas drenadas de mina

\begin{tabular}{cccc}
\hline Universidad & $\begin{array}{c}\text { Nacional de } \\
\text { Huancavelica - UNH* }\end{array}$ & $\begin{array}{c}\text { Nacional Mayor de San } \\
\text { Marcos - UNMSM** }\end{array}$ & $\begin{array}{c}\text { Nacional de Educación “Enrique Guzmán y } \\
\text { Valle” - UNE-EGYV*** }\end{array}$ \\
\hline Documento & $\begin{array}{c}\text { Plan de Estudios 2017- } \\
2021\end{array}$ & $\begin{array}{c}\text { Malla Curricular de Cursos } \\
\text { Comunes }\end{array}$ & $\begin{array}{c}\text { Plan de Estudios de la Carrera Profesional } \\
\text { de Desarrollo Ambiental }\end{array}$ \\
$\begin{array}{c}\text { Semestre/Ciclo de } \\
\text { estudios }\end{array}$ & II Ciclo & I Semestre & I Ciclo \\
Asignatura & Educación Ambiental & $\begin{array}{c}\text { Educación y Desarrollo } \\
\text { Sostenible }\end{array}$ & $\begin{array}{c}\text { Educación Ambiental y Desarrollo } \\
\text { Sostenible }\end{array}$ \\
\hline
\end{tabular}

Fuente: *UNH. Recuperado de http://www.unh.edu.pe/wp-content/uploads/2018/Educacion/Plan_Estudios primaria 2017.pdf ** UNMSM. Recuperado de http://educacion.unmsm.edu.pe/pdf/Plan_2018.pdf

*** UNE. Recuperado de

http://www.une.edu.pe/fan/docs/PLAN\%20DE\%20ESTUDIOS\%20DE\%20DESARROLLO\%20AMBIENTAL.pdf 
De igual manera la universidad debería generar espacios adecuados que permita la interacción con los investigadores y posibilitar medios que difundan a la comunidad universitaria y a la sociedad en general conocer los resultados de las investigaciones mediante materiales didácticos impresos y/o digitales (Carteles educativos, dípticos, fascículos, murales, entre otros).

\section{Conclusiones}

En primer lugar, el estudio ha demostrado que la concentración de Cadmio $(\mathrm{Cd})$ y Plomo $(\mathrm{Pb})$ en el agua drenada de la bocamina Tangana fue de 0,2661 mg Cd/L y 25,7220 mg Pb/L, y excedieron con respecto al nivel de comparación con la categoría 3 del Estándar de Calidad Ambiental (ECA) de agua D.S 004- 2017 MINAM; los resultados de la concentración de cadmio $(\mathrm{Cd})$ y plomo $(\mathrm{Pb})$ en la especie totora fue $0,0007 \mathrm{mg} \mathrm{Cd} / \mathrm{L}$ y es de $0,0037 \mathrm{mg} \mathrm{Pb} / \mathrm{L}$ de la misma manera la cuantificación de concentración de cadmio $(\mathrm{Cd})$ y plomo $(\mathrm{Pb})$ en la especie putacca fue $0,0020 \mathrm{mg} \mathrm{Cd} / \mathrm{L}$ y $0,0948 \mathrm{mg}$ $\mathrm{Pb} / \mathrm{L}$ ). Segundo, la totora (Scirpus californicus) es una especie de planta nativa significativamente bioacumuladora de cadmio $(\mathrm{Cd})$ y plomo $(\mathrm{Pb})$ en la fitorremediación de las aguas drenadas de la mina Tangana. De igual manera, la putacca (Familia apiaceae) es una especie de planta nativa significativamente bioacumuladora de cadmio $(\mathrm{Cd})$ y plomo $(\mathrm{Pb})$ en la fitorremediación de las aguas drenadas de la mina Tangana.

Por ello, Consideramos oportuno y necesario que las currículas de formación de Educación Básica Regular actualicen e inserten contenidos en las áreas curriculares de los diferentes niveles educativos a fin de informar y concientizar a los futuros ciudadanos sobre la existencia de estas especies nativas. En consecuencia, amerita la evaluación y renovación en los planes de estudio de las Universidades públicas y privadas. Se propone a tres universidades nacionales del Perú inserten contenidos relacionados con las especies nativas que capturan metales de aguas drenadas de mina.

\section{Referencias}

Blas, H. D. V., Moreno, O. A. M., \& Huarcaya, A. O. S. (2020). Estudio documental: importancia de la educación ambiental en la educación básica. Revista Iberoamericana Ambiente \& Sustentabilidad, 6-14. http://ambiente-sustentabilidad.org/index.php/revista/article/view/4.

Carpena, R. O., \& Bernal, M. P. (2007). Claves de la fitorremediación: fitotecnologías para la recuperación de suelos. Revista Ecosistemas, 16(2). Recuperado de: https://www.revistaecosistemas.net/index.php/ecosistemas/issue/view/17

Chávez Rodríguez L. (2014). Fitoremediación con especies nativas en suelos contaminados por plomo. Universidad Nacional Agraria La Molina. Facultad de Ciencias. Departamento Académico de 
Ingeniería Ambiental, Física y Meteorología, Lima - Perú. http://repositorio.lamolina.edu.pe/handle/UNALM/2435

El Peruano. (2014). Normas legales. Ley Universitaria 30220. Recuperado de https://busquedas.elperuano.pe/download/full/011B8TsiqIfBx4oqLdeCLI

Dale, E. (1986). El cono de la experiencia. Citado en Teoría y práctica de los medios de enseñanza. Editorial Pueblo y Educación: Ciudad de la Habana.

https://scholar.google.es/scholar?hl=es\&as_sdt=0,5\&q=cono + del + aprendizaje + edgar + dale\#d=gs_cit $\& u=\% 2 F$ scholar\%3Fq\%3Dinfo\%3AaJr90N8zC30J\%3Ascholar.google.com\%2F\%26output $\% 3$ Dcite $\% 26$ scirp $\% 3 \mathrm{D} 2 \% 26 \mathrm{hl} \% 3 \mathrm{Des}$

Delgadillo López A. E., González-Ramírez C. A Prieto-García F., Villagómez Ibarra J. R., AcevedoSandoval O. (2010). Fitorremediación: una alternativa para eliminar la contaminación. Universidad Autónoma del Estado de Hidalgo, Instituto de Ciencias Básicas e Ingeniería, Centro de Investigaciones Químicas, Hidalgo - México. http://www.scielo.org.mx/scielo.php?script=sci_arttext\&pid=S187004622011000200002

MINEDU. Ley General de Educación - Ley N ${ }^{\circ}$ 28044. Recuperado de http://www.minedu.gob.pe/p/ley_general_de_educacion_28044.pdf

MINEDU. (2016). Educación Básica. Programa Curricular de Educación Básica. Recuperado de http://www.minedu.gob.pe/curriculo/pdf/programa-curricular-educacion-inicial.pdf

Núñez-López R.A., Meas-Vong Y, Ortega -Borges R. y Olguín, E. J. (2004). Fitorremediación: fundamentos $\quad y \quad$ aplicaciones, ciencia, México. https://www.revistaciencia.amc.edu.mx/images/revista/55_3/Fitorremediacion.pdf

Ortiz, H., Trejo, R., Valdez, R., Arreola, J., Flores, A., y Lopez, B. (2009). Fitoextracción de plomo y cadmio en suelos contaminados usando quelite (amaranthus hybridus 1.) y micorrizas, Revista Chapingo Serie Horticultura 15(2): México. http://www.scielo.org.mx/scielo.php?script=sci_arttext\&pid=S1027-152X2009000200009

Peña-Salamanca, E. J., Madera-Parra, C. A., Sánchez, J. M., y Medina-Vásquez, J. (2013). Bioprospección de plantas nativas para su uso en procesos de biorremediación: caso helicona psittacorum (heliconiacea). Rev. Acad. Colomb. Cienc. 37 (145): 469-481, 2013. ISSN 03703908. http://www.scielo.org.co/pdf/racefn/v37n145/v37n145a04.pdf

Chaney, Malik, Li, Brown, Brewer, Angle, Baker (1997). Phytoremediation of soil metals. Current Opinion in Biotechnology 8(2), 279-284. https://doi.org/10.1016/S0958-1669(97)80004-3

Rubio, Gutierrez, Martín-Izquierdo, Lozano, Hardisson (2004). El plomo como contaminante 
alimentario. Rev. Toxicol 21:71-80. Recuperado de:

https://www.redalyc.org/pdf/919/91921303.pdf

Uría, M. (2001). Estrategias didáctico-organizativas para mejorar los Centros Educativos. España: Narcea ediciones. https://narceaediciones.es/es/educacion-hoy-estudios/279-estrategiasdidactico-organizativas-para-mejorar-los-centros-educativos-9788427712317.html 